\title{
Proposition of a Schematization Plugin for QGIS
}

\author{
Isadora Barioni $^{\text {a }}$, Luciene Stamato Delazari ${ }^{\mathrm{b}, *}$ \\ ${ }^{a}$ UFPR,Cartographic Engineering Undergraduate, isadorab.barioni@gmail.com \\ ${ }^{b}$ UFPR, Geodetic Sciences Graduate Program, luciene@ufpr.br \\ * Corresponding author
}

Keywords: Schematic Map, GIS, QGIS, Plugin.

\begin{abstract}
:
The Federal University of Paraná (UFPR) has 26 different Campi in several cities in the Parana State, Brazil; in total are 11 million $\mathrm{m}^{2}$ of area, with 500 thousand $\mathrm{m}^{2}$ of constructed area and 316 buildings. UFPR has more than 6000 employees — staff and administrative - about 50,000 undergraduate students and 10,000 graduate students. A great part of this academic community does not know completely the space where they work and study. If we consider the external public who has access to the UFPR these figures are even bigger.
\end{abstract}

The unfamiliarity with space and its characteristics has direct impacts in several issues, such as management of resources (humans and materials), Campi infrastructure management, not only of the exterior but also of the interior of the buildings, security, and other issues that can be supported by the use of geoinformation. From this perspective, we have started a Project named UFPR CampusMap (UCM) whose main goal is to implement a Geographic Information System with information from the indoor and outdoor environments.

Its main purpose is to provide an up-to-date database on the structure of the campus. Among its features is the search and definition of routes between points of interest. The study area is the Campus Centro Politécnico (Curitiba City). In this research we decided to use the cartographic representation in two different aspects: the floor plan that presents the building and rooms and a schematic map, when the visualization scale is bigger, to present the position of a room and the corridors. The schematic representation is also used as a basis for the routing algorithm. Both cartographic representations were derived from a database obtained in a vector file, which details such as doors, windows, stairs, and text. We used QGIS to edit and create the floor plan that has the geometry of the building and rooms.

The schematic map construction is a time consuming task, since involves reducing the complexity of map details, while at the same time preserving the important characteristics (especially topology). In this research, the final goal is to produce a schematic map from floor plans, using a semiautomatic process. In this process, we have used both computational algorithms and manual processes. The schematic map is formed by the transition points-which represent the transition between corridors and rooms, stairs, and bathrooms, for example-and the lines connecting them. The position of the transition points is defined by the following rule: if the transition is a door, the point is placed in the middle of its length, and if the transition is a corridor, for example, a turning point, the point is placed at the end of one line and the beginning of the other. The lines representing the corridors were created manually, using QGIS software, by drawing the central line of the corridor's polygon.

It was verified in this process the need to create a tool to automatize the processes o edition and creation of the schematic map. Thus, we propose the development of a Python QGIS Plugin to perform the schematization process. The plugin consists of four distinct interfaces, each one with one task:

1. First, the centroids of each entrance space (room, for instance) are automatically created;

2. Second, the "skeleton" line of the polygon referring to the corridor layer are created;

3. Third, it is created a line calculated by the smallest distance between the centroid of the room to the centerline generated in the previous step;

4. Fourth, this shorter line and the corridor are joined, turning this layer into a single layer.

Figure 1 presents this process. 



Figure 1. Schematization Process.

After the process of schematization the layer is verified in order to correct topology errors, and to insert the room and corridors attributes. After this, the layer is inserted in a PostGIS database. 